\title{
Differential expression of metabotropic glutamate receptors in the hippocampus and entorhinal cortex of the rat
}

\author{
Majid Fotuhi ${ }^{\text {a }}$, David G. Standaert ${ }^{\text {a,* }}$, Claudia M. Testa ${ }^{\mathrm{a}, \mathrm{b}}$, John B. Penney Jr. ${ }^{\text {a }}$, \\ Anne B. Young ${ }^{\text {a }}$ \\ a Department of Neurology, Massachusetts General Hospital and Harvard Medical School, Fruit St., Boston, MA 02114, USA, \\ ${ }^{b}$ Neuroscience Program, University of Michigan, Ann Arbor, MI 48109, USA
}

(Accepted 20 July 1993)

Key words: Metabotropic glutamate receptor; Hippocampus; Entorhinal cortex; Phosphoinositide-linked receptor; Excitotoxicity

\begin{abstract}
Metabotropic glutamate receptors (mGluRs) have been implicated in a number of hippocampal functions including learning and memory. Five subtypes have been molecularly and pharmacologically characterized. Using in situ hybridization with oligonucleotide probes selective for these five mGluRs, we have found that each has a unique pattern of expression in the hippocampus and entorhinal cortex. mGluR1 is expressed predominantly in the dentate gyrus and CA3. mGluR2 is enriched in the dentate gyrus and inner layer of the entorhinal cortex. mGluR3 is also expressed in these two structures, but unlike all the other mGluRs, is found in white matter areas as well. mGluR4 is present predominantly in CA2 while mGluR5 is concentrated in most regions of the hippocampus and entorhinal cortex. Comparative analysis of the distributions of these receptors with that of the components of their putative downstream signal transduction mechanisms suggests that mGluR5 may be the main subtype of mGluR which mediates the excitatory actions of glutamate in CA1 and could contribute to the elevation of calcium levels found in CA1 pyramidal neurons in long term potentiation and in ischemic/hypoxic injury. mGluR2 and mGluR3, the main subtypes contributing to the inhibitory actions of glutamate, are absent in CA1. Thus, the mGluR-mediated excitatory actions of glutamate can occur in all regions of the hippocampus whereas the mGluR-mediated inhibitory actions of glutamate may be restricted to the dentate gyrus and CA3.
\end{abstract}

\section{INTRODUCTION}

The actions of glutamate, the primary excitatory neurotransmitter in the mammalian brain, are mediated by two types of receptors: ionotropic, which gate ion channels, and metabotropic (mGluR), which are linked via G-proteins to several intracellular signal transduction mechanisms including phosphoinositide (PI) and cyclic AMP systems ${ }^{26}$. mGluRs are believed to play a role in a wide variety of brain functions and seem to be particularly active during long-term processes of neuronal plasticity and remodeling ${ }^{6,34}$. The actions of mGluRs have been studied most extensively in the hippocampus where they appear to participate in learning and the acquisition of memory ${ }^{6,9}$. For example, in a model of spatial learning, glutamate-stimulated PI hydrolysis increases in the hippocampi of rats trained in a radial maze ${ }^{28}$. No such increase is observed in rats trained in a simple shock conditioning

\footnotetext{
* Corresponding author. Fax: (1) (617) 724-1480.
}

avoidance paradigm which does not require spatial learning. Similarly, in a model of long term potentiation (LTP), the PI turnover stimulated by 1-aminocyclopentane-1,3-dicarboxylic acid (ACPD), a specific mGluR agonist, is doubled in the hippocampi of rats which have received tetanic stimulation of the perforant pathway ${ }^{5}$. In in vitro experiments, tetanic stimulation of Schaffer collaterals causes 6-9 fold increases in ACPD-stimulated PI turnover ${ }^{5}$. Finally, the direct application of ACPD in the CA1 region of hippocampal slices induces LTP which is independent of $\mathrm{N}$ methyl-D-aspartate (NMDA) glutamate receptor activation $^{10,11}$. Together these observations suggest that mGluRs play an important role in the neuronal mechanisms underlying the formation and storage of memory.

Until recently, the structures of the mGluRs were unknown, and their biological actions could only be assessed indirectly by observing the effect of glutamate on second messenger systems. Ligand binding studies suggested the existence of several subtypes of mGluRs with heterogeneous distributions ${ }^{12}$, but did not provide sufficient resolution for detailed anatomical 
studies. Within the past two years, molecular cloning techniques have identified five distinct mGluR cDNAs ${ }^{1,18,23,26,37,38}$. Each of these mGluRs has seven putative transmembrane domains and shares structural features with other G-protein-linked receptors. These mGluRs can be classified, based on their pharmacological characteristics and signal transduction mechanisms, into three subgroups ${ }^{26,38}$. In transfected Xenopus oocytes, mGluR1 and mGluR5 receptors (subgroup 1) are linked to the stimulation of PI
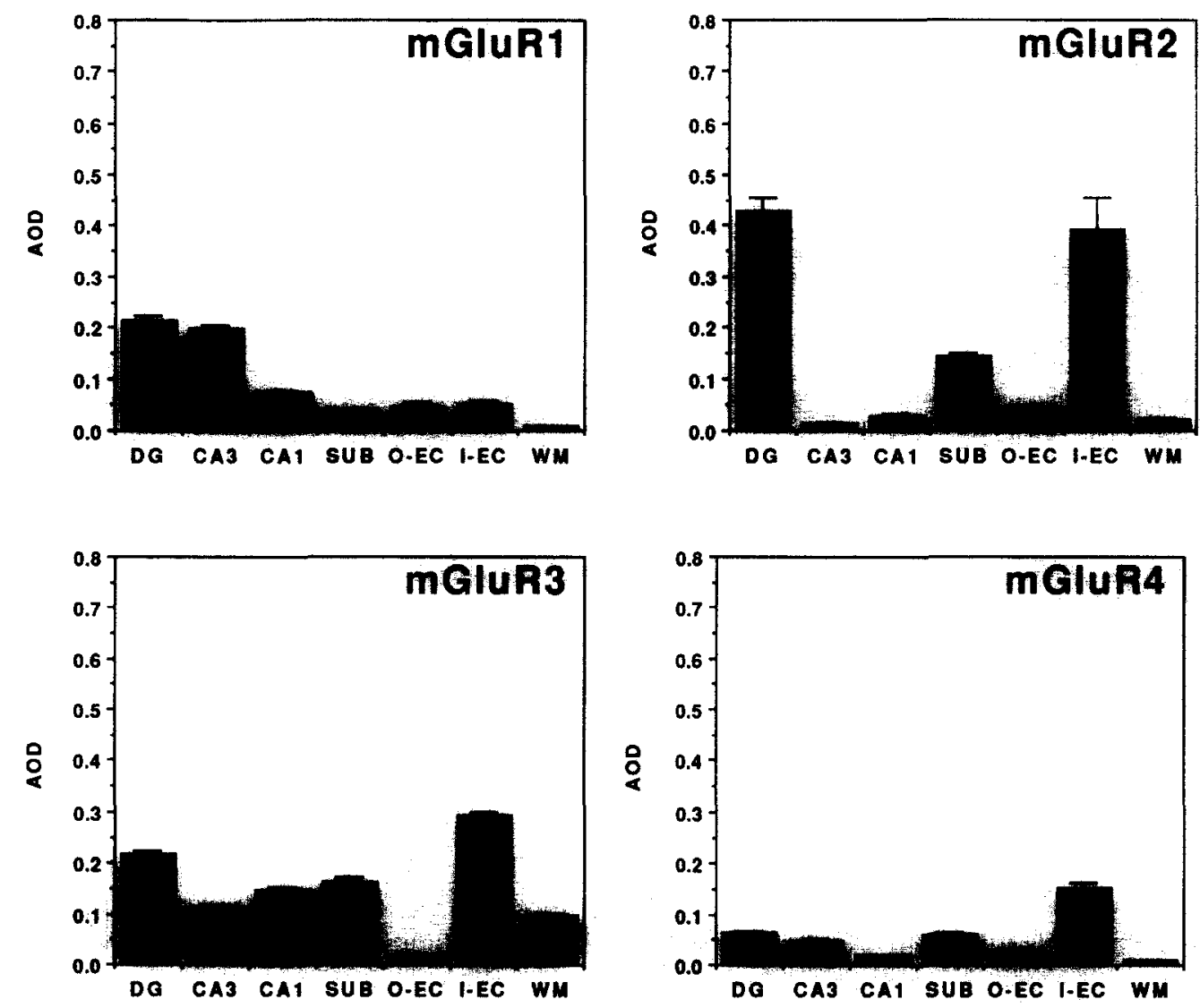

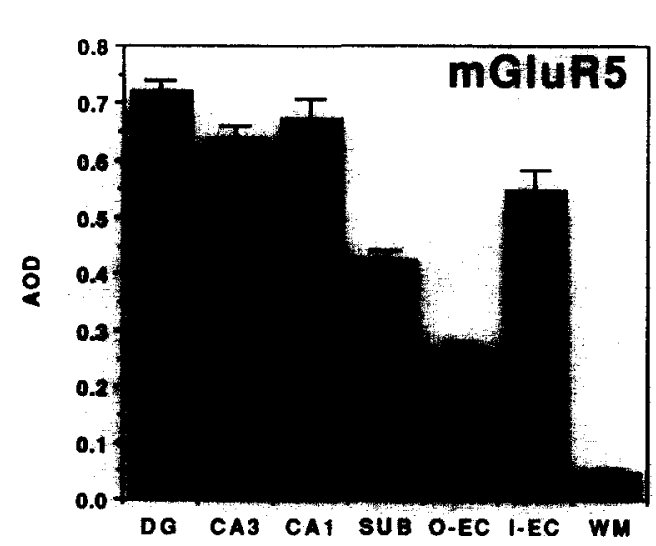

turnover $^{18,23}$. In addition, mGluRl can modulate cAMP and arachidonic acid (AA) production ${ }^{4}$. The activation of mGluR2 and mGluR3 receptors (subgroup 2) by glutamate or ACPD alters forskolin-induced CAMP formation ${ }^{37,38}$. mGluR4 (subgroup 3) is also linked to changes in cAMP levels, but differs from the subgroup 2 receptors in that it is much more sensitive to glutamate than $\mathrm{ACPD}^{26,38}$. Preliminary reports have suggested the existence of two additional mGluRs, one with properties similar to mGluR $4^{26}$ and a second one

Fig. 1. The absolute optical density (AOD) of hybridization signal was measured for mGluR1-5 in adjacent sections processed identically and exposed for the same length of time. Abbreviations are granular layer of the dentate gyrus (DG), pyramidal layer of CA3 (CA3), pyramidal layer of CA1 (CA1), subiculum (SUB), outer entorhinal cortex (O-EC), inner entorhinal cortex (I-EC), and white matter (WM). Data was collected from one side of a total of 9 sections from 3 rats for each probe. The low background level of the film autoradiogram was subtracted from all values. The vertical bars are standard error of mean (S.E.M.) values for each region. 
of unknown pharmacology which is selectively enriched in the olfactory bulb and dentate gyrus ${ }^{33}$.

Considering the significant role of mGluRs in hippocampal function and pathology, the specific localization of these receptors in the hippocampus warrants detailed anatomical examination. In the present study, we have used in situ hybridization with oligodeoxyribonucleotide probes to study the expression of individual mGluRs in the hippocampus and entorhinal cortex of the rat. We found that each of the known mGluRs has a discrete anatomical pattern of expression. Based on the organization of mGluR distributions in the
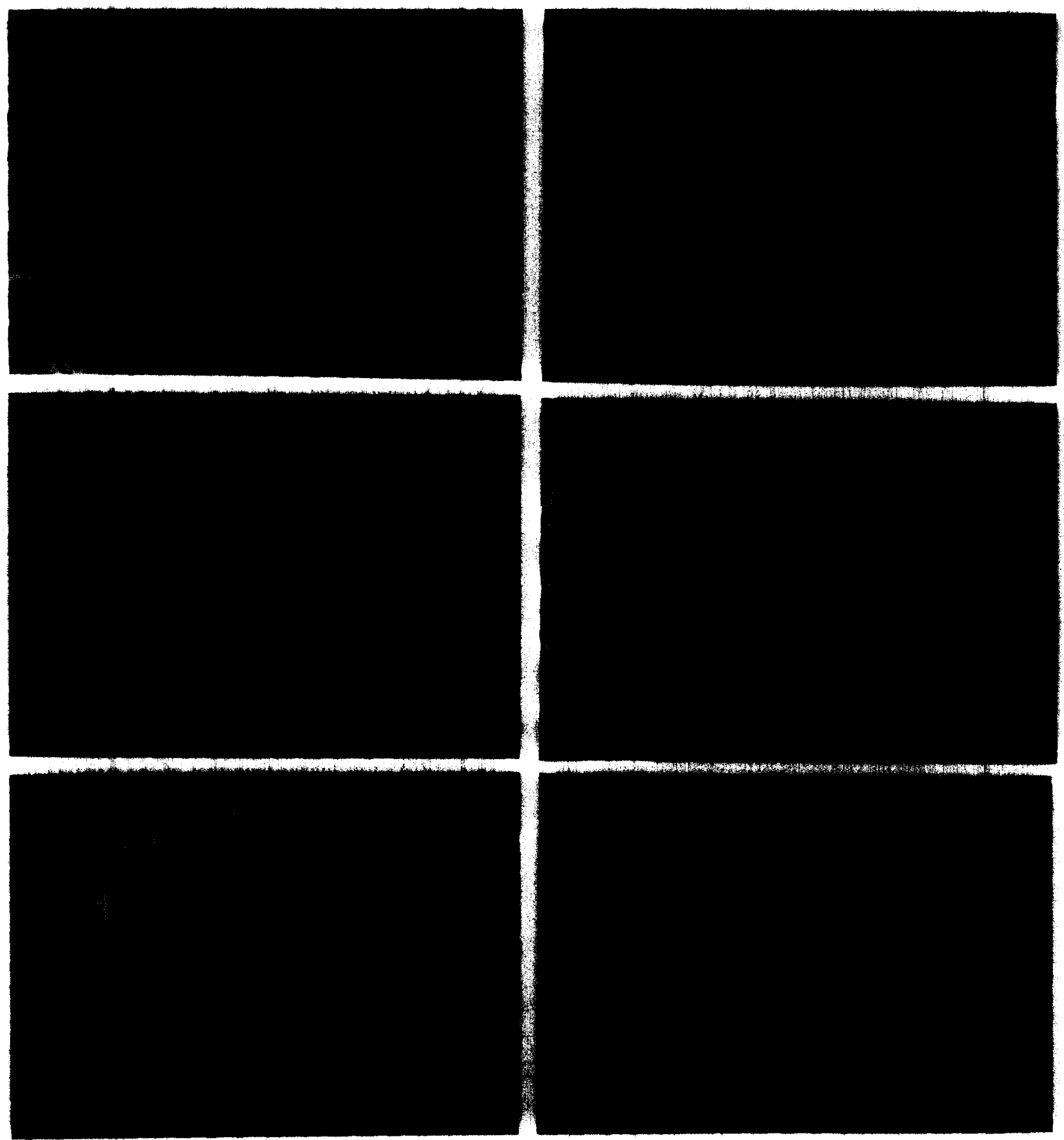

Fig. 2. Dark-field images of hybridization signals of mGluR1 (A), mGluR2 (B), mGluR3 (C), mGluR4 (D), and mGluR5 (E) in the hippocampus and entorhinal cortex. Positive labelling appears white. (F), mGluR5 with excess unlabelled probe. Boxes indicate approximate location of the areas which are shown in Fig. 3. 
hippocampus, we postulate that mGluR5 is the subtype primarily involved in hippocampal excitotoxicity and LTP in CA1, and that the inhibitory actions of glutamate mediated by mGluR2 and mGluR 3 may be limited to the dentate gyrus and CA3.

\section{MATERIALS AND METHODS}

Using published sequence data, oligodeoxyribonucleotide probes for each of the mGluRs were designed to regions of the receptors where homologies between the different mGluRs were minimal. A search through Genebank and EMBL databases using the National Center for Biotechnology Information BLAST network service ${ }^{3}$ revealed no significant homology between selected oligonucleotides and known sequences other than the targeted mGluR. At least two probes were prepared for each receptor. Probes were synthesized using an Applied Biosystems 392 Synthesizer and purified by electrophoresis through a $12 \%$ polyacrylamide gel containing $8 \mathrm{M}$ urea Probes were $3^{\prime}$-end labelled with $\left[{ }^{35} \mathrm{~S}\right] \alpha$-dATP (spec. act: $>1000$ $\mathrm{Ci} / \mathrm{mM}$, Dupont-NEN) by terminal deoxynucleotidyl transferase using a DuPont-NEN kit (NEP 100). Unincorporated label was separated using a sizing column (Stratagene).

Adult male Sprague-Dawley rats were sacrificed by decapitation. Their brains were frozen in isopentane, sectioned $(12 \mu \mathrm{m})$ in a cryostat, mounted on poly-L-lysine-coated slides (Sigma), and stored at $-70^{\circ} \mathrm{C}$. Serially adjacent sets of slides from 6 animals were prepared for this study. Slides were warmed to room temperature (RT), fixed in $4 \%$ paraformaldehyde in $0.1 \mathrm{M}$ phosphate buffer, washed in $0.1 \mathrm{M}$ phosphate buffer saline (PBS), acetylated in $0.1 \mathrm{M}$ triethanolamine ( $\mathrm{pH} 8.0$ ) with $0.25 \%$ acetic anhydride, rinsed again in PBS, and dehydrated through graded ethanol solutions. Slides were incubated overnight at $37^{\circ} \mathrm{C}$ in $60-120 \mu 1$ of hybridization buffer containing $50 \%$ formamide, $0.3 \mathrm{M} \mathrm{NaCl}, 10 \mathrm{mM}$ Tris, $\mathrm{pH} 8.0$, 1 mM EDTA, $10 \%$ dextran sulfate, $1 \times$ Denhart's solution $(0.02 \%$ Ficoll, $0.02 \%$ polyvinylpyrolidone, $0.2 \mathrm{mg} / \mathrm{ml}$ bovine serum albumin, Sigma), $100 \mathrm{mM}$ dithiothreitol, and radiolabelled DNA probes (at an approximate concentration of $30,000 \mathrm{DPM} / \mu \mathrm{l}$ of hybridization buffer). Slides were washed sequentially at RT in $2 \times$ SSC (standard saline citrate: $0.15 \mathrm{M} \mathrm{NaCl}, 0.015 \mathrm{M}$ sodium citrate) for $20 \mathrm{~min}$, $1 \times$ SSC for $10 \mathrm{~min}$, and $0.5 \times \mathrm{SSC}$ for $10 \mathrm{~min}$, at $60^{\circ} \mathrm{C}$ in $0.5 \times \mathrm{SSC}$ for $40 \mathrm{~min}$, and again at RT in $0.5 \times$ SSC for $10 \mathrm{~min}$. Slides were rinsed briefly in $70 \%$ ethanol, air dried, and apposed to $\beta \max$ film (Amersham) for 1 month. Slides were then dipped in NTB-2 emulsion (Kodak) 1:1 with distilled water, exposed for 3 months, developed, counterstained with thionin, coverslipped, and examined under bright- and dark-field optics. Cells which exhibited grain densities more than five times the background were considered labelled. The density of labelling on film autoradiograms was analyzed by a computer-assisted image analysis system (Imaging Research). Intensities of labellings were compared in a one-way repeated measure ANOVA analysis by region with post hoc pairwise comparisons with a significance level of $95 \%$. The specificity of labelling was assessed by addition of a 25 -fold excess of unlabelled probe in the hybridization solution.

\section{RESULTS}

In situ hybridization using probes to the five mGluRs revealed that each receptor has a distinct pattern of expression both within the hippocampus and in other brain regions (Figs. 1 and 2 ). In a preliminary series of experiments, we compared the distribution of labelling obtained using probes to at least two different regions of each receptor. In each case, we found that the patterns of labelling were identical, though the absolute intensity of labelling varied. Based on these experiments, we selected one probe for each receptor for detailed analysis (Table 1). Specificity of the probes was confirmed by the absence of hybridization signal when a 25-fold excess of unlabelled oligodeoxyribonucleotide was included in the hybridization solution (Fig. 2F).

\section{$m G l u R 1$}

The intensity of mGluR1 labelling in the hippocampus was highest in the granular layer of the dentate gyrus and the pyramidal layer of CA3 (Figs. $1 \mathrm{~A}$ and 2A). CA1 pyramidal cells also contained significant amounts of hybridization signal compared to the adjacent white matter areas, but, consistent with previous reports $^{16,22,36}$, this labelling was less intense than that in the pyramidal cells of CA3. Unlike all other mGluRs, mGluR1 was expressed in scattered neurons in the dorsal segment of striatum oriens of CAl (Fig. 3A). Low but significant mGluR1 labelling was observed in neuronal cell bodies in the subiculum and entorhinal cortex. Outside the hippocampus, prominent mGluR 1 labelling was seen over the Purkinje cell layer of the cerebellum and to a lesser extent in the striatum. The intensity of the hippocampal mGluR1 labelling in the granular layer of the dentate gyrus and the pyramidal layer of CA3 was higher than that of the striatum but was lower than that in the Purkinje cells of cerebellum (Fig. 1A; and Testa et al., in preparation). mGluR1 labelling in the brain areas examined was primarily neuronal and did not appear in white matter regions (Figs. $1 \mathrm{~A}$ and $2 \mathrm{~A}$ ).

TABLE I

The sequence of oligonucleotide probes used for detection of $m G l u R l-5$ and the region of $m G l u R s$ to which they correspond

\begin{tabular}{lll}
\hline$m$ GluR & Amino acids & Sequence $\left(5^{\prime}-3^{\prime}\right)$ \\
\hline mGluR1 & $484-498$ & CCC CAC GTG GAC ATA GTC ATA GCG ATT AGC TTC TGT GTA CTG CAG \\
mGluR2 & $123-137$ & GGT GAC AGC TGT AGG AGC ATC ACT GTG GGT GGC ATA GGA GCC ATC \\
mGluR3 & $841-855$ & CTG AGA ATA GGT GGT TGC AGT TCC GCT GAC GCT GAA CCT GTT GAC \\
mGluR4 & $881-892$ & GGT CTC CAG GTT CTC ACA CAG CTC TGA TTT GGC TTC CCC ATT GGG \\
mGluR5 & $124-138$ & GGA GCG GAA GGA AGA AGA TCC ATC TAC ACA GCG TAC CAA ACC TTC \\
\hline
\end{tabular}



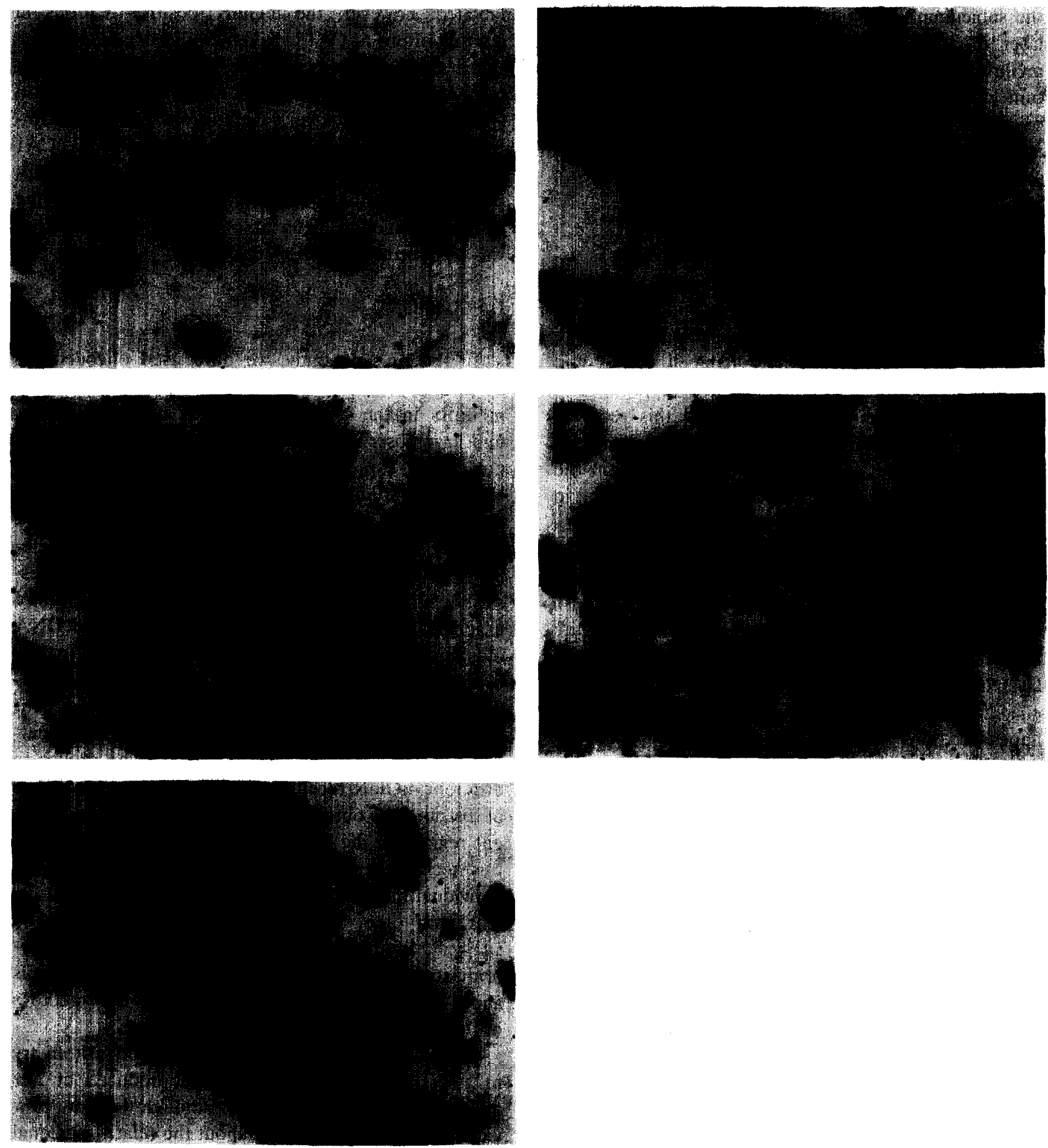

Fig. 3. High magnification of hybridization signals in the hippocampus (marked with boxes in Fig. 2) with probes for mGluR1(A), mGluR2(B), mGluR3(C), mGluR4(D), and mGluR5(E). mGluR1 is found in a subgroup of neurons in the dorsal segment of stratum oriens of CA1 (A) where no other mGluR was detected. mGluR2 was present only in neuronal cell bodies of dentate gyrus (B). However, mGluR3 was found in both neurons and glia (arrow heads) of the dentate gyrus (C). mGluR4 was detected in some pyramidal cells in CA2 (D). In contrast, mGluR5 was expressed at high levels in all areas of the hippocampus and entorhinal cortex including CA1 (E). Bar $=30 \mu \mathrm{m}$.

\section{mGluR2}

Hybridization with probes to mGluR2 revealed a striking differential pattern of neuronal labelling in the hippocampus (Figs. 1B, 2B, and 3B). The granular cells of the dentate gyrus were intensely labelled (Fig. 3B), while pyramidal cells in CA3/CA1 lacked significant labelling above the adjacent white matter areas. A moderate level of mGluR2 expression was present in 
the subiculum. The neuronal cell bodies in the inner layer of entorhinal cortex contained high levels of mGluR2, while those in the outer layer lacked significant labelling. Outside the hippocampus, mGluR2 hybridization signal was present in the cerebral cortex and cerebellum. No glial mGluR2 labelling was observed in any of the brain areas examined.

\section{$m$ GluR3}

In contrast to all other mGluRs, mGluR3 was expressed in both grey and white matter regions (Figs. $1 \mathrm{C}, 2 \mathrm{C}$ and $3 \mathrm{C}$ ). Within the hippocampus, mGluR3 labelling was most intense in the granular layer of the dentate gyrus where both neuronal cell bodies and small, densely-stained cells with scant cytoplasm, most likely representative of glia, expressed this receptor (Fig. 3C). The mGluR3 labelling in the pyramidal layer of CA3 was at the same level as the labelling in the adjacent white matter areas. However, the labelling in the pyramidal layer of CA1 and in the subiculum was significantly greater than the white matter areas. On film autoradiograms, the mGluR3 labelling in the inner layer of the entorhinal cortex appeared more intense than that either in the outer layer or in the white matter areas. On emulsion-dipped sections, the inner layer labelling appeared in both neurons and putative glia. The low mGluR3 labelling in the outer layer appeared only glial. Outside of the hippocampus, mGluR3 was observed in many grey and white matter structures, but was particularly prominent in the reticular nucleus of the thalamus (Fig. 2C; and Testa et al., in preparation).

\section{$m$ GluR4}

mGluR4 labelling in the hippocampus was unique as only the pyramidal cells of the CA2 area were strongly labelled (Figs. 1D, 2D and 3D). The dentate gyrus, CA3, and subiculum expressed mGluR4 at low but detectable levels. No positive labelling could be detected in CA1. A low but significant level of signal was also observed in neurons along the inner layer of the entorhinal cortex. In contrast to the low abundance in the hippocampal areas, strong mGluR4 labelling was observed in other regions of the brain, particularly in the cerebellar granular layer and the thalamus. As with mGluR1 and mGluR2, mGluR4 labelling was not detected in glia.

\section{$m$ GluR5}

mGluR5 labelling was abundant throughout the hippocampus (Figs. $1 \mathrm{E}$ and $2 \mathrm{E}$ ). The granule cells of the dentate gyrus, the subiculum, and the pyramidal layer neurons in CA3 and CA1 (Fig. 3F) were all strongly labelled; however, the mGluRl-positive neurons in stratum oriens of CA1 lacked mGluR5 expression. The outer and inner layers of the entorhinal cortex both contained neurons with high mGluR5 hybridization signals. The inner layer labelling was particularly in tense and appeared comparable to the CA.3/CAl pyramidal cell labelling (Fig. 2E). Outside the hippocampus, mGluR5 labelling was prominent in the striatum while no detectable mGluR5 hybridization was observed in the cerebellar cortex.

\section{DISCUSSION}

In the present study, we have used oligodeoxyribonucleotide probes to study the expression of five mGluRs in the hippocampus and entorhinal cortex, and have observed that each mGluR exhibits a unique pattern of expression. Several previous in situ hy bridization studies have described the expression of one or more of the mGluRs in the brain $23,29,36,37,38$ Each of these previous studies employed RNA riboprobes which offer high sensitivity but may not necessarily discriminate between closely related members of a gene family ${ }^{21}$. We have employed a panel of DNA oligonucleotide probes designed to minimize cross-hybridization between the mGluRs. We have also examined the patterns of hybridization in near-adjacent sections to maximize comparability. The unique patterns of expression we have observed using probes to different $\mathrm{mGluR}$ receptors as well as the concordance of distributions obtained with multiple probes to different regions of the same mGluR support the ability of this technique to selectively label the mRNA's for individual mGluRs. Our findings are generally consistent with other studies employing Northern analysis $^{18,37,38}$, immunohistochemistry ${ }^{22,16}$, and in situ hybridization $23,36,37,38,29,38,16$, and provide further details about the regional and cellular distribution of mGluRs in the hippocampus and entorhinal cortex.

In addition, we have used quantitative densitometry to assess the intensity of labelling produced by our mGluR probes in these areas. Although this data provides important information about the relative regional abundance of mGluR mRNA's and serves as a foundation for further studies on the regulation of expression of these genes, several points need to be considered. First, the specific activity of probes which are enzymatically 3 '-end labelled may vary somewhat between experiments. In our experience this variation is relatively small. Second, although the densities of the film autoradiograms are closely related to the amount of ${ }^{35} \mathrm{~S}$ present ${ }^{25}$, different regions of the brain have varying densities of neuronal perikarya. Finally, when compar- 
ing the signals produced by dissimilar probes, the signal may be affected not only by the specific activity of the probes, but also by differences in efficiency of hybridization and differential preservation of mRNAs ${ }^{42}$; both of these factors may also affect Northern analysis. Thus, although the signals obtained from different probes may not be strictly proportional to the absolute amount of the mGluR mRNA's present, the high reproducibility of the distributions and comparability to data obtained using techniques such as Northern analysis ${ }^{18,37,38}$ suggest that they are a reasonable relative measure of the expression of these genes.

We observed that each area of the hippocampus and entorhinal cortex expresses a distinctive combination of mGluRs. Dentate gyrus is the only structure which expresses all mGluRs. In this area mGluR1, mGluR2, and mGluR5 are present at high levels, while mGluR3 and mGluR4 are detectable only at modest to low levels. CA3 is rich in mGluR1 and mGluR5, low in mGluR4 and nearly void of mGluR2. CA2 is the only area of the hippocampus with substantial expression of mGluR4. CA2 also contains mGluR1 and mGluR5. The CA1 pyramidal layer is predominantly enriched in mGluR5, has low levels of mGluR1 and mGluR3, and lacks any significant level of mGluR2 or mGluR4. The subiculum expresses high levels of mGluR5, moderate levels of mGluR2, especially along the outer margin, and only low levels of mGluR1 and mGluR4. The inner and outer layers of the entorhinal cortex exhibit differential expression of the mGluRs. While both layers express only small amounts of mGluR1, the mGluR2 and mGluR3 hybridization signals were 8- to 10 -fold higher in the inner layer than in the outer layer. mGluR5 labelling was also higher in the inner layer.

\section{Distribution of $m G$ GluR expression in relation to ligand binding sites}

Ligand binding studies with $\left[{ }^{3} \mathrm{H}\right] \mathrm{glutamate}$ have suggested the presence of two distinct types of mGluR binding sites in the brain ${ }^{12}$. The type $1 \mathrm{mGluR}$ binding sites (met 1), which share the pharmacology of mGluR1 and mGluR5, are widely distributed in the brain and are enriched in the entorhinal cortex, molecular layer of dentate gyrus, stratum oriens of CA3 and CA1, septum, and especially in the molecular layer of the cerebellum. Type 2 mGluR binding sites (met 2) which share the pharmacology of mGluR2 and mGluR3, overlap with type 1 sites and are found in the molecular layer of dentate gyrus, stratum moleculare of CA3, stratum oriens and stratum radiatum of CA1 as well as in the outer layer of cortex, striatum, and thalamus. Comparison of the pharmacology and distribution of these sites with the expression of mGluRs in the hippocampus suggests that in the dentate gyrus, $\mathrm{CA} 3$, and CA1 mGluR1 and mGluR5 may collectively contribute to met 1 , while in the dentate gyrus and CA3, mGluR2 and mGluR3 may together account for the met $2^{12}$. The correlation of mGluR1/mGluR5 with met 1 and mGluR2/mGluR3 with met 2 occurs in the majority of other brain areas. An exception is the presence of met 2 in CA1 where no mGluR2/mGluR3 mRNA could be detected. This may be due to the presence of a yet unidentified form of mGluR in CA1 or to presynaptic met 2 receptors on Schaffer collateral terminals.

\section{Distribution of mGluRs in relation to the components of the signal transduction cascades}

The ability of the mGluRs to contribute to various hippocampal functions requires not only the expression of the appropriate receptor subtypes but also the presence of the specific components of signal transduction mechanisms down stream of the receptor. For example, both mGluR1 and mGluR5 are believed to be linked to the PI signalling system ${ }^{26}$. The activation of PI-linked receptors results in the hydrolysis of phosphotidyl inositol bisphosphate into diacylglycerol (DAG) and inositol triphosphate $\left(\mathrm{IP}_{3}\right)^{8}$, each having a distinct physiological activity and specific distribution in the brain ${ }^{39}$. DAG activates protein kinase $\mathrm{C}$, while $\mathrm{IP}_{3}$ binds to the $\mathrm{IP}_{3}$ receptor and mediates the release of calcium from intracellular stores. $\left[{ }^{3} \mathrm{H}\right] \mathrm{Phorbol}$ ester binding studies indicate that the DAG limb of PI turnover is present in all areas of hippocampus, with the highest amounts in $\mathrm{CA} 1^{40}$. $\left[{ }^{3} \mathrm{H}\right] \mathrm{IP}_{3}$ binding studies ${ }^{41}$ and immunohistochemical studies ${ }^{16}$ in turn show that $\mathrm{IP}_{3}$ receptor levels are high in CA1 and low in dentate gyrus and CA3. Comparing these localizations with the expression of genes for mGluR1 and mGluR5 in the hippocampus suggests that mGluR1 stimulation may activate the DAG/PKC branch of the PI pathway while mGluR5 stimulation could activate both the DAG / PKC branch as well as the $\mathrm{IP}_{3}$ receptor branch, as $\mathrm{IP}_{3}$ receptor is enriched in CA1 where only mGluR5 is found in high levels. Thus, the simultaneous activation of mGluR1 and mGluR5 in the hippocampus by glutamate is likely to lead to an increase in intracellular calcium levels in the CA1 area, where both mGluR5 and $\mathrm{IP}_{3}$ receptors are present, but not in dentate gyrus and $\mathrm{CA} 3$ where the $\mathrm{IP}_{3}$ receptor levels are low.

Unlike the differential localization of PI-linked mGluRs and the components of the PI cascade, the putative cAMP-linked mGluRs and the components of adenylate cyclase cascade appear in the same regions of the hippocampus. mGluR1, mGluR2, and mGluR3 are all enriched in dentate gyrus or CA3, and are low 
to undetectable in CA1. Similarly, adenylate cyclase, as depicted by $\left[{ }^{3} \mathrm{H}\right]$ forskolin binding, is enriched in dentate gyrus and $\mathrm{CA} 3$ and is absent in $\mathrm{CA}^{39}$. Since the dentate gyrus largely projects to $\mathrm{CA} 3$, adenylate cyclase in CA3 may mediate the post-synaptic actions of mGluR1 expressed by CA3 neurons and/or the presynaptic actions of mGluR2 or mGluR 3 expressed by dentate gyrus neurons in their mossy fiber terminals.

\section{Distribution of $m G$ GuRs in relation to LTP}

Recent reports provide increasing evidence for the critical role of mGluRs in LTP $^{9,17,27}$. LTP induction depends on increases in intracellular calcium levels, which are believed to be mediated at least in part by NMDA receptors ${ }^{9}$. mGluRs may participate in the induction and maintenance of LTP not only by enhancing the NMDA receptor's effects but also by exerting their own effects independent of NMDA receptor activation. Antagonists of mGluRs, L-2-amino-3-phosphonopropionate (AP3) and 2-amino-4-phosphonobutyric acid (AP4), can block the induction and reduce the duration of LTP in hippocampal slice preparations ${ }^{20,30}$ Moreover, the specific mGluR agonist, ACPD, can augment tetanus-induced potentiation and enable subthreshold amounts of NMDA or low-frequency stimuli to induce $\mathrm{LTP}^{14,24,31}$. Recently, it has been shown that ACPD can induce LTP in the CA1 region of the hippocampus by itself, independent of activation of NMDA receptors ${ }^{10,11}$.

Either mGluR1 or mGluR5 subtypes could mediate the elevations in calcium levels required for LTP in duction and maintenance. We have observed that the primary $m$ GluR in the $C A 1$ region, where $A C P D$ induces LTP, is mGluR5. Others have noted that pertussis toxin, which inactivates certain G-proteins, fails to block tetanus-induced LTP in the CA1 region in vitro ${ }^{19}$. Interestingly, mGluR5 differs from mGluR1 in that its actions are not sensitive to pertussis toxin'. These observations lead us to propose that it is mGluR5, linked to the $\mathrm{IP}_{3}$ receptor (through a pertussis-toxin insensitive G-protein), which mediates the PI-mediated calcium fluxes required for the induction of LTP in CA1.

Distribution of $m$ GluRs in relation to the inhibition of neurotransmission by glutamate

Although glutamate is believed to be the major excitatory neurotransmitter in the brain, it may also inhibit neurotransmission at certain synapses ${ }^{26}$. mGlu$\mathrm{R} 2,3$, and 4 , which are linked to reductions in cAMP levels, can potentially mediate such inhibitory actions of glutamate. AP4, a relatively selective agonist of mGluR4, and ACPD, an agonist of mGluR2 and
mGluR3, depress excitatory monosynaptic transmission in hippocampal neurons in culture ${ }^{7} .15$. In these experiments, the AP4 suppression of synaptic neurotransmis sion was similar to that caused by GABA and baclofen and was shown to be mediated by a presynaptic receptor $^{15}$. We observed a virtual absence of mGluR4 in the hippocampus suggesting that mGluR2 and/or mGluR3 may be the primary mediators of the inhibitory actions of glutamate in the hippocampus.

Distribution of $m G$ GluRs in relation to neurotoxicity in the hippocampus

In addition to their physiological actions in learning and memory, mGluRs may also play a role in the neuronal injury which occurs in the hippocampus after ischemia or hypoxia ${ }^{2}$. Following carotid occlusion in rats, the glutamate-stimulated PI turnover in the hippocampus increases up to 6 -fold ${ }^{35}$. The CAl region exhibits a greater vulnerability to neuronal injury ${ }^{13}$. In an in vitro model of hypoxia, the application of the mGluR antagonist AP3 during hypoxia reduces the extent of injury as measured by evoked field potentials in the CA1 region of the hippocampus by about 80 $100 \%{ }^{30}$. Moreover, in an in vivo model, direct injection of the agonist ACPD into the hippocampus leads to seizures and selective damage of neurons in the dentate gyrus and CA1 region ${ }^{32}$.

As for LTP, either mGluR1 or mGluR5 can contribute to the elevation of calcium levels required for excitotoxity. Neurons in CA1 express large amounts of mGluR5 and $\mathrm{IP}_{3}$ receptor suggesting that mGluR5 stimulation could raise cytosolic calcium levels to the threshold for toxicity in these cells. The CA3 neurons which are less vulnerable also are enriched in the PI-linked receptors mGluR1 and mGluR5 but are practically devoid of $\mathrm{IP}_{3}$ receptor. Thus, the coupling of the PI-linked mGluR5 and $\mathrm{IP}_{3}$ receptors in CAl and the lack of such coupling in CA3 may account for the selective sensitivity of CAl neurons to ischemic injury.

\section{Conclusions}

Metabotropic glutamate receptors have been implicated in a large number of hippocampal functions. The pattern of expression of the mGluRs in the hippocampus provides clues as to the subtypes of mGluRs which contribute to such functions. mGluR5 is the primary mGluR in the CA1 region and may be the receptor which mediates the increases in calcium levels required either for physiological hippocampal functions such as long term enhancements of synaptic efficacy or for damaging CA1 neurons in ischemia. mGluR2 and mGluR3 may mediate the glutamate-stimulated inhibition of synaptic neurotransmission in the hippocampus. 
The comparison of the distribution of PI-linked mGluRs, mGluR1 and mGluR5, and the components of the PI cascade raises the possibility that the same mGluR may be preferentially linked to different branches of the PI system in different areas of hippocampus, and that this differential linkage may account for the relative vulnerability of CA1 neurons to ischemic injury.

Acknowledgements. We thank M.V. Catania, G.B. Landwehrmeyer, and Z.R. Hollingsworth for their technical assistance and fruitful discussions. Supported by USPHS grants NS 19613 and AG 08671. D.G.S. is a Howard Hughes Medical Institute Physician Research Fellow and recipient of an American Academy of Neurology Research Fellowship Award in Neuropharmacology. CMT is a University of Michigan Medical Scientist Training Program fellow.

\section{REFERENCES}

1 Abe, T., Sugihara, H., Nawa, H., Shigemoto, R., Mizuno, N. and Nakanishi, S., Molecular characterization of a novel metabotropic glutamate receptor mGluR5 coupled to inositol phosphate $/ \mathrm{Ca}^{2+}$ signal transduction, J. Biol. Chem., 267 (1992) 13361-13368.

2 Aleppo, G., Pisani, A., Copani, A., Bruno, V., Aronica, E., D'Agata, V., Canonico, P.L. and Nicoletti, F., Metabotropic glutamate receptors and neuronal toxicity. In E.A. Bazan (Ed.), Neurobiolgoy of Essential Fatty Acids, Plenum, New York, 1992, pp. 137-142.

3 Altschul, S., Stephen, F., Gish, W., Miller, W., Myers, E. and Lipman, D., Basic local alignment search tool, J. Mol. Biol., 215 (1990) 403-410.

4 Aramori, I. and Nakanishi, S., Signal transduction and pharmacological characteristics of a metabotropic glutamate receptor, mGluR1, in tranfected CHO cells, Neuron, 8 (1992) 757-765.

5 Aronica, E., Frey, U., Wagner, M., Schroeder, H., Krug, M., Ruthrich, H., Catania, M.V., Nicoletti, F. and Reymann, K.G., Enhanced sensitivity of "metabotropic" glutamate receptors after induction of long-term potentiation in rat hippocampus, $J$. Neurochem., 57 (1991) 376-383.

6 Baskys, A., Metabotropic receptors and 'slow' excitatory actions of glutamate agonists in the hippocampus, Trends Neurosci, 15 (1992) 92-96.

7 Baskys, A. and Malenka, R.C., Agonists at metabotropic glutamate receptors presynaptically inhibit EPSC's in neonatal rat hippocampus, J. Physiol., 444 (1991) 687-701.

8 Berridge, M.J., Inositol triphosphate and calcium signalling, $\mathrm{Na}$ ture, 361 (1993) 315-322.

9 Bliss, T.V.P. and Collingridge, G.L., A synaptic model of memory: long-term potentiation in the hippocampus, Nature, 361 (1993) 31-38.

10 Bortolotto, Z.A. and Collingridge, G.L., Activation of glutamate metabotropic receptors induces long-term potentiation, Eur. $J$. Pharmacol., 214 (1992) 297-8.

11 Bortolotto, Z.A. and Collingridge, G.L., Characterization of LTP induced by the activation of glutamate metabotropic receptors in area CA1 of the hippocampus, Neuropharmacology, 32 (1993) $1-9$.

12 Catania, M.V., Hollingsworth, Z., Penney, J.B. and Young, A.B., Quisqualate resolves two distinct metabotropic $\left[{ }^{3} \mathrm{H}\right] \mathrm{glutamate}$ binding sites, NeuroReport, 4 (1993) 311-313.

13 Choi, D.W. and Rothman, S.M., The role of glutamate neurotoxicity in hypoxic-ischemic neuronal death, Annu. Rev. Neurosci., 13 (1990) 171-82.

14 Collingridge, G.L., Harvey, J., Frenguelli, B.G., Bortolotto, Z.A., Bashir, Z.I. and Davies, C.H., Amino acid receptors and long-term potentiation: targets for the development of cognitive enhancers, Int. Acad. Biomed. Res., 2 (1992) 41-49.
15 Forsythe, I.D. and Clements, J.D., Presynaptic glutamate receptors depress excitatory monosynaptic transmission between mouse hippocampal neurones, J. Physiol., 429 (1990) 1-16.

16 Fotuhi, M., Sharp, A.H., Glatt, F.G., Hwang, P.M., Krosigk M.V., Snyder, S.H. and Dawson, T.M., Differential localization of phosphoinositide-linked metabotropic glutamate receptor (mGluR1) and the inositol 1,4,5-triphosphate receptor in rat brain, J. Neurosci., 13 (1993) 2001-2012.

17 Herrero, I., Miras-Portugal, M.T. and Sanchez-Prieto, J., Positive feedback of glutamate exocytosis by metabotropic presynaptic receptor stimulation, Nature, 360 (1992) 163-166.

18 Houamed, K.M., Kuijper, J.L., Gilbert, T.L., Haldeman, B.A., O'Hara, P.J., Mulvihill, E.R., Almers, W. and Hagen, F.S. Cloning, expression and gene structure of a $G$ protein-coupled glutamate receptor from rat brain, Science, 252 (1991) 1318-1321.

19 Ito, I., Okada, D. and Sugiyama, H., Pertussis toxin suppresses long-term potentiation of hippocampal mossy fiber synapses, Neurosci. Let., 90 (1988) 181-85.

20 Izumi, Y., Clifford, D.B. and Zorumski, C.F., 2-Amino-3-phosphonopropionate blocks the induction and maintenance of longterm potentiation in rat hippocampal slices, Neurosci. Let., 122 (1991) 187-190.

21 Lewis, M.E. and Baldino, F., Probes for in situ hybridization histochemistry. In M.F. Chesselet (Ed.), In Situ Hybridization Histochemistry, CRC, Boston, 1990, pp. 1-21.

22 Martin, L.J., Blackstone, C.D., Huganir, R.L. and Price, D.L., Cellular localization of a metabotropic glutamate receptor in rat brain, Neuron, 9 (1992) 259-70.

23 Masu, M., Tanabe, Y., Tsuchida, K., Shigemoto, R. and Nakanishi, S., Sequence and expression of a metabotropic glutamate receptor, Nature, 349 (1991) 760-765.

24 McGuinness, N., Anwyl, R. and Rowan, M., Trans-ACPD en hances long-term potentiation in the hippocampus, Eur. J. Pharmacol., 197 (1991) 231-232.

25 Miller, J.A., The calibration of ${ }^{35} \mathrm{~S}$ or ${ }^{32} \mathrm{P}$ with ${ }^{14} \mathrm{C}$-labeled brain paste or ${ }^{14} \mathrm{C}$-plastic standards for quantitative autoradiography using LKB Ultrofilm or Amersham Hyperfilm, Neurosci. Lett., 121 (1991) 211-214.

26 Nakanishi, S., Molecular diversity of glutamate receptors and implications for brain function, Science, 258 (1992) 597-603.

27 Nicholls, D.G., A retrograde step forward, Nature, 360 (1992) $106-7$.

28 Nicoletti, F., Valerio, C., Pellegrino, F., Drago, F., Scapagnini, U. and Canonico, P.L., Spatial learning potentiates the stimulation of phosphoinositide hydrolysis by excitatory amino acids in rat hippocampal slices, J. Neurochem., 51 (1988) 725-729.

29 Ohishi, H., Shigemoto, R., Nakanishi, S. and Mizuno, N., Distribution of the messenger RNA for a metabotropic glutamate receptor, mGluR2, in the central nervous system of the rat, Neuroscience, 53 (1993) 1009-1018.

30 Opitz, T. and Reymann, K.G., Blockade of metabotropic glutamate receptors protects rat CA1 neurons from hypoxic injury, NeuroReport, 2 (1991) 455-7.

31 Radpour, S. and Thomson, A.M., Synaptic enhancement induced by NMDA and QP receptors and presynaptic activity, Neurosci. Lett., 138 (1992) 119-122.

32 Sacaan, A.I., Monn, J.A. and Schoepp, D.D., Intrastriatal injection of a selective metabotropic excitatory amino acid receptor agonist induces contralateral turning in the rat, J. Pharmacol. Exp. Ther., 259 (1991) 1366-70.

33 Saugstad, J.A., Segerson, T.P., Mulvihill, E.R. and Westbrook, G.L., Isolation of novel metabotropic glutamate receptor cDNA's from rat olfactory bulb, Soc. Neurosci. Abstr., 18 (1992) 1363.

34 Schoepp, D., Bockaert, J. and Sladeczek, F., Pharmacological and functional characteristics of metabotropic excitatory amino acid receptors, Trends Pharmacol. Sci., 11 (1990) 508-515.

35 Seren, M.S., Aldinio, C., Zanoni, A., Leon, A. and Nicoletti, F. Stimulation of inositol phospholipid hydrolysis by excitatory amino acids is enhanced in brains slices from vulnerable regions after transient global ischemia, J. Neurochem., 53 (1989) 1700-1705.

36 Shigemoto, R., Nakanishi, S. and Mizuno, N., Distribution of the mRNA for a metabotropic glutamate receptor (mGluR1) in the 
central nervous system: an in situ hybridization study in adult and developing rat, J. Comp. Neurol., 322 (1992) 121-135.

37 Tanabe, Y., Masu, M., Ishii, T., Shigemoto, R. and Nakanishi, S. A family of metabotropic glutamate receptors. Neuron. 8 (1992) $169-179$

38 Tanabe, Y.. Nomura, A., Masu, M., Shigemoto, R., Mizuno, N and Nakanishi, S., Signal transduction, pharmacologic properties and expression patterns of two rat metabotropic glutamate recep tors, mGluR3 and mGluR4, J. Neurosci. 13 (1993) 1372 1378.

39 Worley. P.F., Baraban, J.M., Colvin, J.S. and Snyder, S.H., Inositol trisphosphate receptor localization in brain: variable stoichiometry with protein kinase C. Nature, 325 (1987) 159-161
40 Worley, P.F.. Baraban, J.M. and Snyder, S.H., Heterogenous localization of protein kinase $C$ in rat brain antoradiographi analysis of phorbol ester receptor binding. /. Vermosec, 6 (1486 $199-207$

41 Worley, P.F., Baraban, J.M. and Snyder, S.H., Inositol 1,4,5-1ri phosphate receptor binding: autoradiographic localization in rat brain, J. Neurosci., 9 (1989) 339-346.

42 Young. W.S., III and Kuhar, M.J., Quantitative in situ hybridiza tion and determination of mRNA content. In G.R. Uhl (Ed.), in Situ Hybridization in Brain, Plenum. New York. 1986. pp. 243. 